\title{
Stabilization effect of distributed delay with strong kernel in three-neuron BAM neural networks
}

\author{
S. Mandal ${ }^{1 *}$, P. D. Gupta ${ }^{2}$, A. B. Roy ${ }^{2}$, N. C. Majee ${ }^{2}$ \\ ${ }^{1}$ Department of Mathematics, JIS College of Engineering, Kalyani 741235, India \\ ${ }^{2}$ Department of Mathematics, Jadavpur University, Kolkata 700032, India \\ *Corresponding author E-mail: santanu_mndl@yahoo.co.in
}

Copyright (C2014 S. Mandal et. al. This is an open access article distributed under the Creative Commons Attribution License, which permits unrestricted use, distribution, and reproduction in any medium, provided the original work is properly cited.

\begin{abstract}
The effect of distributed delays with strong kernel in the dynamics in three-neuron BAM neural network model is studied. Instead of destabilization phenomena this neural systems become asymptotically stable through Hopf bifurcation with the gradual increment of mean delay. Existence of Hopf bifurcation is studied in frequency domain. Direction and stability of Hopf bifurcating periodic solutions are analyzed using Nyquist criterion and graphical Hopf bifurcation theorem.
\end{abstract}

Keywords: Distributed delay, Hopf bifurcation, Frequency domain, Stabilization effect, Strong kernel

\section{Introduction}

Neural networks ususally have spatial extent due to presence of multitude of parallel pathways with varity of axon sizes and lengths. As a result distribution of propagation of delays occur. Generally distributed delys was considered in the models of poplulation biology and epidemic system. Recently it is observed that distributed delays are considered in neural network system. Some of these research works studied how distributed delays affect the dynamics differently from discrete delays. Atay[1,2] studied a system consisting of two simple oscillation with gap junctional coupling by incorporating a uniform distribution of delays. He observed that it is easier to destroy oscillations with a distribution of delays than with a discrete delay. Jirsa and Ding [3] analyzed an $\mathrm{n}$ dimentional linear system with linear decay and arbitrary connections with common delay. They showed that the stability region of the trivial solution for any distribution of delays is larger thanthat of discrete delay. Eurich et al.[4] considered the effect of distributed delays in Predator-Prey models and ecological food webs. Generally the occurrence of delays in population dynamics is usually regarded a destabilizing factor leading to the extinction of species. They demonstrated complementarily that delay distribution yield larger stability regimes than that of single delay. Meyer et al.[5] quantified the distribution of delays in the avian isthmotectal feed back loop. By investigating a mathematical model of coupled neurons with distributed delays which enhance the stability of the system. We also observed the stabilizing effect arrises from the contribution of smaller delays introduced through the delay distribution. Zhang et al.[6] considered a Lotka-Volterra two species predator-prey system with a distributed delay. In their study They found the positive equilibrium of the system is always locally asymptotically stable. They also observed that at the positive equilibrium the system under goes the Hopf bifurcation when the average time delay in the delay kernel crosses certain critical values.

From above discussions one can easily conclude that a system with a distribution of delays is inherently more 
stable than the same system with a discrete delay. In this paper we observed stabilization effect on neural network system in presence of weak and strong kernel. The weak kernel qualitatively indicates that the maximum weighted response of the growth rate is due to current population density. That is past densities have decreasing influence. The strong kernel means that the maximum influence on growth rate responses for the past time [7].

The following three-neuron BAM neural network model with distributed delay having self connections under dynamic threshold is considered.

$$
\begin{aligned}
d x_{1}(t) / d t & =-x_{1}(t)+\beta_{1}^{\prime} f\left[x_{1}(t)-\gamma \int_{-\infty}^{0} F(s) x_{1}(t-s) d s-c_{1}\right] \\
& +b^{\prime} f\left[x_{2}(t)-\gamma \int_{-\infty}^{0} F(s) x_{2}(t-s) d s-c_{2}\right] \\
& +b^{\prime} f\left[x_{3}(t)-\gamma \int_{-\infty}^{0} F(s) x_{3}(t-s) d s-c_{3}\right] \\
d x_{2}(t) / d t & =-x_{2}(t)+a^{\prime} f\left[x_{1}(t)-\gamma \int_{-\infty}^{0} F(s) x_{1}(t-s) d s-c_{1}\right] \\
& +\beta^{\prime} f\left[x_{2}(t)-\gamma \int_{-\infty}^{0} F(s) x_{2}(t-s) d s-c_{2}\right] \\
d x_{3}(t) / d t & =-x_{3}(t)+a^{\prime} f\left[x_{1}(t)-\gamma \int_{-\infty}^{0} F(s) x_{1}(t-s) d s-c_{1}\right] \\
& +\beta^{\prime} f\left[x_{3}(t)-\gamma \int_{-\infty}^{0} F(s) x_{3}(t-s) d s-c_{3}\right]
\end{aligned}
$$

where, $x_{i}(i=1,2,3)$ denotes the mean soma potential of the neuron i. The non-negative constant $\beta^{\prime}$, corresponds to the strength of neurons to itself. $b^{\prime}$ corresponds to the strength of neuron 2 and 3 on neuron 1 . $a^{\prime}$ represents the strength of neuron 1 on neuron 2 and 3. $\gamma \neq 0$ is the measure of the inhibitory influence of the past history. $c_{i}(i=1,2,3)>0$ denotes the neuronal threshold. The term $x_{i}$ in the argument of function $\mathrm{f}$ represents local positive feedback. The weight function $\mathrm{F}(\mathrm{s})$ is a non-negative bounded function defined on $(0, \infty)$ to reflect the influence of the past states on the current dynamics. $\mathrm{F}(\mathrm{s})$ is called the delay kernel. Here $\mathrm{F}(\mathrm{s})$ is taken as strong kernel in the form $F(s)=\mu^{2} s e^{-\mu s}, s \in(0, \infty), \mu>0$.

Here we studied stability criteria of the system (1) in frequency domain. The frequency domain approach was initiated and developed by Allwright[8], Mees and Chua [9] and Moiola and Chen[10]. There are many advantages of frequency domain approach relative to time domain. A typical one is its pictorial characteristic that utilizes advanced computer graphical capabilities. Using this approach we showed that system become stable when the system can undergo a Hopf bifurcation when the mean delay $\mu$ crosses certain critical value. Sufficient conditions are obtained for existence of Hopf bifurcation. The stability of the bifurcating periodic solutions are determined by the Nyquist criterion[11] and the graphical Hopf bifurcation theorem[10]. Lastly we discussed about larger stability region for strong kernel than that for weak kernel.

The paper is organised as follows: In section 2, we derived sufficient conditions are derived for the existence of Hopf bifurcation in the frequency domain. In section 3, the stability of Hopf bifurcating periodic solutions are means of Nyquist criterion and the graphical Hopf bifurcation theorem. Simulation results with figures are presented in sec. 4 to justify the analytical results obtained. It appears from simulation results that an enlarged stability regime is obtained for strong kernel in comparison to weak kernel. Finally conclusions are drawn in section 5. 


\section{Existence of Hopf bifurcation}

By using frequency domain technique we are going to deduce sufficient conditions for existence of Hopf bifurcation. Let us assume that

(H1) $f \in C^{4}(R), f(0)=0$, and $\mu f(\mu)>0$ for $\mu \neq 0$.

The general form of delay kernel $F(s)$ is as follows:

$$
F(s)=\mu^{n+1} \frac{s^{n} e^{-\mu s}}{n !}, \quad n=0,1,2 .
$$

where $\mu$ is a parameter denoting the rate of decay of the effects of past memories and it is a positive real number. It is also known as exponentially fading memory.

$n=0$ represents weak kernel whereas $n=1$ represents strong kernel.

For simplicity let us set $c_{1}=c_{2}=c_{3}=0$, and let

$$
\begin{aligned}
& x_{1}(t)=y_{1}(t)-\gamma \int_{0}^{\infty} F(s) y_{1}(t-s) d s \\
& x_{2}(t)=y_{2}(t)-\gamma \int_{0}^{\infty} F(s) y_{2}(t-s) d s \\
& x_{3}(t)=y_{3}(t)-\gamma \int_{0}^{\infty} F(s) y_{3}(t-s) d s
\end{aligned}
$$

Then (1) assumes the following form:

$$
\begin{aligned}
& \frac{d x_{1}}{d t}=-x_{1}(t)+\beta^{\prime} f\left[x_{1}(t)\right]-\beta^{\prime} \gamma \int_{0}^{\infty} F(-s) f\left[x_{1}(t+s)\right] d s \\
& +b^{\prime} f\left[x_{2}(t)\right]-b^{\prime} \gamma \int_{0}^{\infty} F(-s) f\left[x_{2}(t+s)\right] d s+b^{\prime} f\left[x_{3}(t)\right] \\
& -b^{\prime} \gamma \int_{0}^{\infty} F(-s) f\left[x_{3}(t+s)\right] d s \\
& \frac{d x_{2}}{d t}=-x_{2}(t)+a^{\prime} f\left[x_{1}(t)\right]-a^{\prime} \gamma \int_{0}^{\infty} F(-s) f\left[x_{1}(t+s)\right] d s \\
& +\beta^{\prime} f\left[x_{2}(t)\right]-\beta^{\prime} \gamma \int_{0}^{\infty} F(-s) f\left[x_{2}(t+s)\right] d s \\
& \frac{d x_{3}}{d t}=-x_{3}(t)+a^{\prime} f\left[x_{1}(t)\right]-a^{\prime} \gamma \int_{0}^{\infty} F(-s) f\left[x_{1}(t+s)\right] d s \\
& +\beta^{\prime} f\left[x_{3}(t)\right]-\beta^{\prime} \gamma \int_{0}^{\infty} F(-s) f\left[x_{3}(t+s)\right] d s
\end{aligned}
$$

Since

$$
F(r)=\mu^{2} r e^{-\mu r}
$$

, we have

$$
\begin{gathered}
\int_{-\infty}^{0} F(-r) f[x(t+r)] d r=\int_{-\infty}^{0} \mu^{2}(t-s) e^{\mu(s-t)} f[x(s)] d s \\
=\mu^{2} e^{-\mu t}\left\{t \int_{-\infty}^{t} e^{\mu s} f[x(s)] d s-\int_{-\infty}^{t} s e^{\mu s} f[x(s)] d s\right\}
\end{gathered}
$$


Taking derivative on both sides of (4) and using (5) we get

$$
\begin{gathered}
\frac{d^{2} x_{1}}{d t^{2}}=-\frac{d x_{1}}{d t}+\beta^{\prime} f^{\prime}\left(x_{1}\right) \frac{d x_{1}}{d t}+b^{\prime} f^{\prime}\left(x_{2}\right) \frac{d x_{2}}{d t}+b^{\prime} f^{\prime}\left(x_{3}\right) \frac{d x_{3}}{d t} \\
-\mu\left[\frac{d x_{1}}{d t}+x_{1}-\beta^{\prime} f\left(x_{1}\right)-b^{\prime} f\left(x_{2}\right)-b^{\prime} f\left(x_{3}\right)\right] \\
-\beta^{\prime} \gamma \mu^{2} e^{-\mu t} \int_{-\infty}^{t} e^{\mu s} f\left(x_{1}\right) d s-b^{\prime} \gamma \mu^{2} e^{-\mu t} \int_{-\infty}^{t} e^{\mu s}\left[f\left(x_{2}\right)+f\left(x_{3}\right)\right] d s \\
\frac{d^{2} x_{2}}{d t^{2}}=-\frac{d x_{2}}{d t}+a^{\prime} f^{\prime}\left(x_{1}\right) \frac{d x_{1}}{d t}+b^{\prime} f^{\prime}\left(x_{2}\right) \frac{d x_{2}}{d t}-\mu\left[\frac{d x_{2}}{d t}+x_{2}-a^{\prime} f\left(x_{1}\right)-b^{\prime} f\left(x_{2}\right)\right] \\
\frac{d^{2} x_{3}}{d t^{2}}=-\frac{d x_{3}}{d t}+a^{\prime} \mu^{2} e^{-\mu t} \int_{-\infty}^{t} e^{\mu s} f\left(x_{1}\right) \frac{d x_{1}}{d t}+b^{\prime} f^{\prime}\left(x_{3}\right) \frac{d x_{3}}{d t}-\mu\left[\frac{d x_{3}}{d t}+b_{3}^{\prime}-\mu^{2} e^{-\mu t} \int_{-\infty}^{t} e^{\mu s} f\left(x_{2}\right) d s\right. \\
\left.-a^{\prime} \gamma \mu^{2} e^{-\mu t} \int_{-\infty}^{t} e^{\mu s} f\left(x_{1}\right) d s-b^{\prime} \gamma \mu^{2} e^{-\mu t} \int_{-\infty}^{t} e^{\mu s} f\left(x_{3}\right)\right]
\end{gathered}
$$

Taking derivative again on both sides and setting $\quad x_{4}=\frac{d x_{1}}{d t} \quad, \quad x_{5}=\frac{d x_{2}}{d t} \quad, \quad x_{6}=\frac{d x_{3}}{d t} \quad, \quad x_{7}=\frac{d^{2} x_{1}}{d t^{2}}, x_{8}=$ $\frac{d^{2} x_{2}}{d t^{2}}, x_{9}=\frac{d^{2} x_{3}}{d t^{2}}$ we get the following set of ordinary differential equations

$$
\begin{gathered}
\frac{d x_{1}}{d t}=x_{4} \\
\frac{d x_{2}}{d t}=x_{5} \\
\frac{d x_{3}}{d t}=x_{6} \\
\frac{d x_{4}}{d t}=x_{7} \\
\frac{d x_{5}}{d t}=x_{8} \\
\frac{d x_{6}}{d t}=x_{9} \\
\frac{d x_{7}}{d t}=-\mu^{2} x_{1}-\left(\mu^{2}+2 \mu\right) x_{4}-(2 \mu+1) x_{7}+b^{\prime}(1-\gamma) \mu^{2}\left[f\left(x_{2}\right)+f\left(x_{3}\right)\right]+b^{\prime}(1-\gamma) \mu^{2} f\left(x_{1}\right) \\
+2 \mu b^{\prime}\left[f^{\prime}\left(x_{2}\right) x_{5}+f^{\prime}\left(x_{3}\right) x_{6}\right]+2 \mu b^{\prime} f^{\prime}\left(x_{1}\right) x_{4}+b^{\prime}\left[f^{\prime}\left(x_{2}\right) x_{8}+f^{\prime}\left(x_{3}\right) x_{9}\right]+b^{\prime} f^{\prime}\left(x_{1}\right) x_{7} \\
+b^{\prime}\left[f^{\prime \prime}\left(x_{2}\right) x_{5}^{2}+f^{\prime \prime}\left(x_{3}\right) x_{6}^{2}\right]+b^{\prime} f^{\prime \prime}\left(x_{1}\right) x_{4}^{2} \\
\frac{d x_{8}}{d t}=-\mu^{2} x_{2}-\left(\mu^{2}+2 \mu\right) x_{5}-(2 \mu+1) x_{8}+a^{\prime}(1-\gamma) \mu^{2} f\left(x_{1}\right)+b^{\prime}(1-\gamma) \mu^{2} f\left(x_{2}\right)+2 \mu a^{\prime} f^{\prime}\left(x_{1}\right) x_{4} \\
f^{2} x_{3}-\left(\mu_{2}\right) x_{5}+a^{\prime} f^{\prime}\left(x_{1}\right) x_{7}+b^{\prime} f^{\prime}\left(x_{2}\right) x_{8}+a^{\prime} f^{\prime \prime}\left(x_{1}\right) x_{4}^{2}+b^{\prime} f^{\prime \prime}\left(x_{2}\right) x_{5}^{2} \\
\end{gathered}
$$




$$
+2 \mu b^{\prime} f^{\prime}\left(x_{3}\right) x_{6}+a^{\prime} f^{\prime}\left(x_{1}\right) x_{7}+b^{\prime} f^{\prime}\left(x_{3}\right) x_{9}+a^{\prime} f^{\prime \prime}\left(x_{1}\right) x_{4}^{2}+b^{\prime} f^{\prime \prime}\left(x_{3}\right) x_{6}^{2}
$$

(7) can be written as

$$
\frac{d x}{d t}=A(\mu) x+H(x)
$$

where $\quad x=\left(x_{1}, x_{2}, x_{3}, \ldots, x_{9}\right)^{T}$.

$$
\begin{aligned}
& A(\mu)=\left[\begin{array}{ccccccccc}
0 & 0 & 0 & 1 & 0 & 0 & 0 & 0 & 0 \\
0 & 0 & 0 & 0 & 1 & 0 & 0 & 0 & 0 \\
0 & 0 & 0 & 0 & 0 & 1 & 0 & 0 & 0 \\
0 & 0 & 0 & 0 & 0 & 0 & 1 & 0 & 0 \\
0 & 0 & 0 & 0 & 0 & 0 & 0 & 1 & 0 \\
0 & 0 & 0 & 0 & 0 & 0 & 0 & 0 & 1 \\
-\mu^{2} & 0 & 0 & -\left(\mu^{2}+2 \mu\right) & 0 & 0 & -(2 \mu+1) & 0 & 0 \\
0 & -\mu^{2} & 0 & 0 & -\left(\mu^{2}+2 \mu\right) & 0 & 0 & -(2 \mu+1) & 0 \\
0 & 0 & -\mu^{2} & 0 & 0 & -\left(\mu^{2}+2 \mu\right) & 0 & 0 & -(2 \mu+1)
\end{array}\right]
\end{aligned}
$$

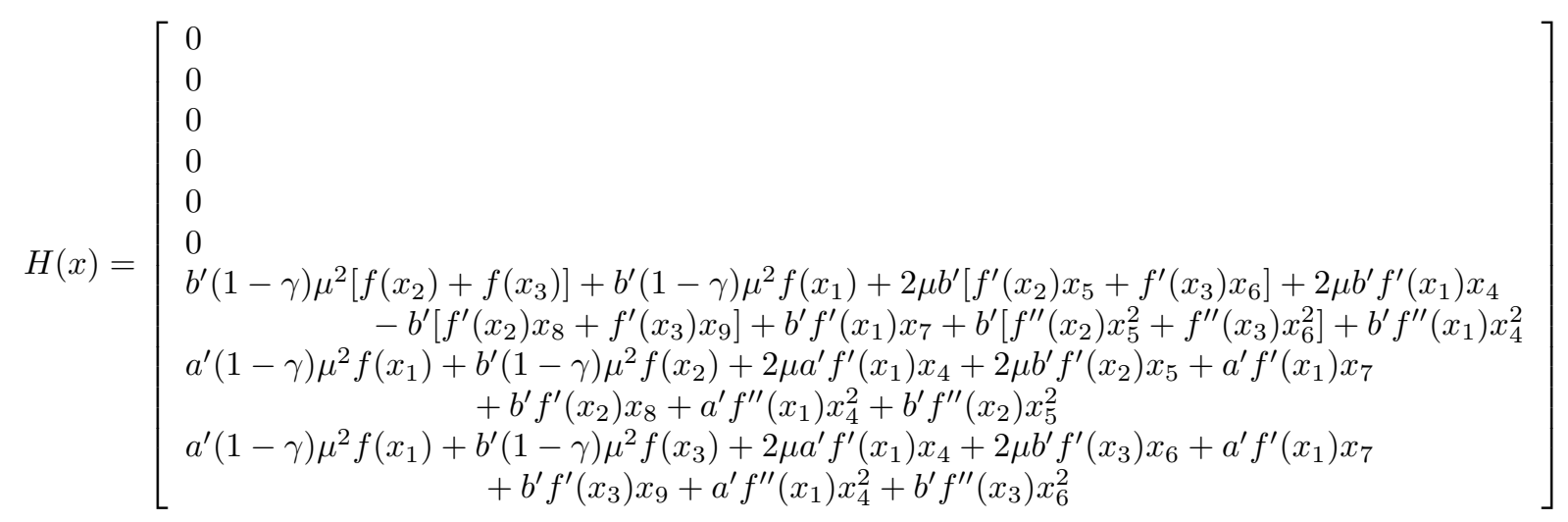

By introducing a 'state-feedback control' $u=g(y ; \mu)$ we obtain the following linear system with a non-linear feedback,

$$
\left.\begin{array}{l}
\frac{d x}{d t}=A(\mu) x+B u \\
y=-C x \\
u=g(y ; \mu)
\end{array}\right]
$$

where

$$
B=\left[\begin{array}{lll}
0 & 0 & 0 \\
0 & 0 & 0 \\
0 & 0 & 0 \\
0 & 0 & 0 \\
0 & 0 & 0 \\
0 & 0 & 0 \\
1 & 0 & 0 \\
0 & 1 & 0 \\
0 & 0 & 1
\end{array}\right] \quad ; \quad C=I .
$$

and

$$
\begin{gathered}
u=g(y ; \mu)= \\
{\left[\begin{array}{l}
\left(b^{\prime}(1-\gamma) \mu^{2}\left[f\left(-y_{2}\right)+f\left(-y_{3}\right)\right]+b^{\prime}(1-\gamma) \mu^{2} f\left(-y_{1}\right)-2 \mu b^{\prime}\left[f^{\prime}\left(-y_{2}\right) y_{5}+f^{\prime}\left(-y_{3}\right) y_{6}\right]\right. \\
-2 \mu b^{\prime} f^{\prime}\left(-y_{1}\right) y_{4}-b^{\prime}\left[f^{\prime}\left(-y_{2}\right) y_{8}+f^{\prime}\left(-y_{3}\right) y_{9}\right]-b^{\prime} f^{\prime}\left(-y_{1}\right) y_{7}+b^{\prime}\left[f^{\prime \prime}\left(-y_{2}\right) y_{5}^{2}+f^{\prime \prime}\left(-y_{3}\right) y_{6}^{2}\right] \\
\left.+b^{\prime} f^{\prime \prime}\left(-y_{1}\right) y_{4}^{2}\right) \\
\left(a^{\prime}(1-\gamma) \mu^{2} f\left(-y_{1}\right)+b^{\prime}(1-\gamma) \mu^{2} f\left(-y_{2}\right)-2 \mu a^{\prime} f^{\prime}\left(y_{1}\right) y_{4}-2 \mu b^{\prime} f^{\prime}\left(-y_{2}\right) y_{5}-a^{\prime} f^{\prime}\left(-y_{1}\right) y_{7}\right. \\
\left.-b^{\prime} f^{\prime}\left(-y_{2}\right) y_{8}+a^{\prime} f^{\prime \prime}\left(-y_{1}\right) y_{4}^{2}+b^{\prime} f^{\prime \prime}\left(-y_{2}\right) y_{5}^{2}\right) \\
\left(a^{\prime}(1-\gamma) \mu^{2} f\left(-y_{1}\right)+b^{\prime}(1-\gamma) \mu^{2} f\left(-y_{3}\right)+2 \mu a^{\prime} f^{\prime}\left(-y_{1}\right) y_{4}-2 \mu b^{\prime} f^{\prime}\left(-y_{3}\right) y_{6}-a^{\prime} f^{\prime}\left(-y_{1}\right) y_{7}\right. \\
\left.-b^{\prime} f^{\prime}\left(-y_{3}\right) y_{9}+a^{\prime} f^{\prime \prime}\left(-y_{1}\right) y_{4}^{2}+b^{\prime} f^{\prime \prime}\left(-y_{3}\right) y_{6}^{2}\right)
\end{array}\right]}
\end{gathered}
$$


Next taking a Laplace Transform on equation (10) we have a standard transfer matrix of the linear part as follows

$$
\begin{aligned}
& G(s ; \mu)=c[s I-A(\mu)]^{-1} B \\
& =\left[\begin{array}{ccccccccc}
s & 0 & 0 & -1 & 0 & 0 & 0 & 0 & 0 \\
0 & s & 0 & 0 & -1 & 0 & 0 & 0 & 0 \\
0 & 0 & s & 0 & 0 & -1 & 0 & 0 & 0 \\
0 & 0 & 0 & s & 0 & 0 & -1 & 0 & 0 \\
0 & 0 & 0 & 0 & s & 0 & 0 & -1 & 0 \\
0 & 0 & 0 & 0 & 0 & s & 0 & 0 & -1 \\
\mu^{2} & 0 & 0 & \left(\mu^{2}+2 \mu\right) & 0 & 0 & (s+2 \mu+1) & 0 & 0 \\
0 & \mu^{2} & 0 & 0 & \left(\mu^{2}+2 \mu\right) & 0 & 0 & (s+2 \mu+1) & 0 \\
0 & 0 & \mu^{2} & 0 & 0 & \left(\mu^{2}+2 \mu\right) & 0 & 0 & (s+2 \mu+1)
\end{array}\right] \\
& {\left[\begin{array}{ccc}
0 & 0 & 0 \\
0 & 0 & 0 \\
0 & 0 & 0 \\
0 & 0 & 0 \\
0 & 0 & 0 \\
0 & 0 & 0 \\
1 & 0 & 0 \\
0 & 1 & 0 \\
0 & 0 & 1
\end{array}\right]=\frac{1}{(1+s)\left(s+\mu^{2}\right)}\left[\begin{array}{ccc}
1 & 0 & 0 \\
0 & 1 & 0 \\
0 & 0 & 1 \\
s & 0 & 0 \\
0 & s & 0 \\
0 & 0 & s \\
s^{2} & 0 & 0 \\
0 & s^{2} & 0 \\
0 & 0 & s^{2}
\end{array}\right]}
\end{aligned}
$$

To this end, if this feedback system is linearized about the equilibrium $y=0$, then the Jacobian of (11) at origin is given by

$$
\begin{gathered}
J(\mu)=\left.\frac{\partial g}{\partial y}\right|_{y=0}= \\
{\left[\begin{array}{ccccccccc}
-b(1-\gamma) \mu^{2} & -b(1-\gamma) \mu^{2} & -b(1-\gamma) \mu^{2} & -2 \mu \beta & -2 \mu b & -2 \mu b & -\beta & -b & -b \\
-a(1-\gamma) \mu^{2} & -b(1-\gamma) \mu^{2} & 0 & -2 \mu a & -2 \mu b & 0 & -a & -b & 0 \\
-a(1-\gamma) \mu^{2} & 0 & -b(1-\gamma) \mu^{2} & -2 \mu a & 0 & -2 \mu b & -a & 0 & -b
\end{array}\right]}
\end{gathered}
$$

where, $\quad a=a^{\prime} f^{\prime}(0) \quad, \quad b=b^{\prime} f^{\prime}(0) \quad, \quad b=b^{\prime} f^{\prime}(0)$

So we have,

$$
\begin{gathered}
\multicolumn{1}{c}{G(s ; \mu) J(\mu)=\frac{1}{(1+s)\left(s+\mu^{2}\right)} \times} \\
{\left[\begin{array}{ccccccccc}
-b(1-\gamma) \mu^{2} & -b(1-\gamma) \mu^{2} & -b(1-\gamma) \mu^{2} & -2 \mu \beta & -2 \mu b & -2 \mu b & -\beta & -b & -b \\
-a(1-\gamma) \mu^{2} & -b(1-\gamma) \mu^{2} & 0 & -2 \mu a & -2 \mu b & 0 & -a & -b & 0 \\
-a(1-\gamma) \mu^{2} & 0 & -b(1-\gamma) \mu^{2} & -2 \mu a & 0 & -2 \mu b & -a & 0 & -b \\
-b s(1-\gamma) \mu^{2} & -b s(1-\gamma) \mu^{2} & -b s(1-\gamma) \mu^{2} & -2 \mu \beta s & -2 \mu b s & -2 \mu b s & -\beta s & -b s & -b s \\
-a s(1-\gamma) \mu^{2} & -b s(1-\gamma) \mu^{2} & 0 & -2 \mu a s & -2 \mu b s & 0 & -a s & -b s & 0 \\
-a s(1-\gamma) \mu^{2} & 0 & -b s(1-\gamma) \mu^{2} & -2 \mu a s & 0 & -2 \mu b s & -a s & 0 & -b s \\
-b s^{2}(1-\gamma) \mu^{2} & -b s^{2}(1-\gamma) \mu^{2} & -b s^{2}(1-\gamma) \mu^{2} & -2 \mu \beta s^{2} & -2 \mu b s^{2} & -2 \mu b s^{2} & -\beta s^{2} & -b s^{2} & -b s^{2} \\
-a s^{2}(1-\gamma) \mu^{2} & -b s^{2}(1-\gamma) \mu^{2} & 0 & -2 \mu a s^{2} & -2 \mu b s^{2} & 0 & -a s^{2} & -b s^{2} & 0 \\
-a s^{2}(1-\gamma) \mu^{2} & 0 & -b s^{2}(1-\gamma) \mu^{2} & -2 \mu a s^{2} & 0 & -2 \mu b s^{2} & -a s^{2} & 0 & -b s^{2}
\end{array}\right]}
\end{gathered}
$$

Now let us set

$$
h(\lambda, s ; \mu)=\operatorname{det}|\lambda I-G(s ; \mu) J(\mu)|=0
$$

Then by applying the generalized Nyquist stability criterion with $s=i \omega$, the following results can be established.

\section{Lemma 1}

(Moila and Chen, 1996[10]) If an eigen value of the corresponding Jacobian of the non-linear system, in the time domain, assumes a purely imaginary value $i \omega_{0}$ at a particular $\mu=\mu_{0}$, then the corresponding eigen value of the constant matrix $\left[G\left(i \omega_{0} ; \mu_{0}\right) J\left(\mu_{0}\right)\right]$ in the frequency domain must assume the value $-1+i 0$ at $\mu=\mu_{0}$. 
Let us consider the case $b=0, \gamma=1$.

To apply Lemma 1 , let $\widehat{\lambda}=\widehat{\lambda}(i \omega, \mu)$ be the eigen value of $[G(i \omega, \mu) J(\mu)]$, that satisfies $\hat{\lambda}\left(i \omega_{0}, \mu_{0}\right)=-1+i 0$. Then

$$
\begin{gathered}
h\left(-1, i \omega_{0} ; \mu_{0}\right)_{(\beta=0, \gamma=1)}=\frac{2 a b\left[\left(\mu_{0}+i \omega_{0}\right)^{2}-\mu_{0}^{2}\right]^{2}}{\left(1+i \omega_{0}\right)^{2}\left(\mu_{0}+i \omega_{0}\right)^{4}}-1=0 \\
\Rightarrow 2 a b\left[\left(\mu_{0}+i \omega_{0}\right)^{2}-\mu_{0}^{2}\right]^{2}=\left(1+i \omega_{0}\right)^{2}\left(\mu_{0}+i \omega_{0}\right)^{4}
\end{gathered}
$$

Separating real and complex parts of (16) we get

$$
\begin{gathered}
\omega^{6}+m_{1} \omega^{4}+m_{2} \omega^{2}+m_{3}=0 \\
m_{4} \omega^{4}+m_{5} \omega^{2}+m_{6}=0
\end{gathered}
$$

where,

$$
\begin{aligned}
& m_{1}=(2 a b-1)-2 \mu(4+3 \mu) \\
& m_{2}=\mu^{2}[6-8 a b+\mu(8+\mu)] \\
& m_{3}=-\mu^{4} \\
& m_{4}=(1+2 \mu) \\
& m_{5}=2 \mu\left[2 a b-1-3 \mu-\mu^{2}\right] \\
& m_{6}=\mu^{3}(2+\mu)
\end{aligned}
$$

Eliminating $\omega$ from (17) and (18) we have

$$
\begin{gathered}
\left(m_{2}^{2} n_{4}^{2}-2 m_{1} m_{3} m_{4}^{2}-m_{1} m_{2} m_{4} m_{5}+3 m_{3} m_{4} m_{5}+m_{2} m_{5}^{2}\right) m_{6}+\left(m_{1}^{2} m_{4}-2 m_{2} m_{4}-m_{1} m_{5}\right) m_{6}^{2}+m_{6}^{3} \\
-m_{3}\left(-m_{3} m_{4}^{2}+m_{2} m_{4}^{2} m_{5}-m_{1} m_{4} m_{5}^{2}+m_{5}^{2}\right)=0 \\
\Rightarrow c_{0}+c_{1} \mu+c_{2} \mu^{2}+\ldots+c_{7} \mu^{7}+c_{8} \mu^{8}=0 \equiv g(\mu)
\end{gathered}
$$

where,

$$
\begin{aligned}
& c_{0}=96(-0.5+a b)^{3} \\
& c_{1}=-96+464 a b-512 a b^{2} \\
& c_{2}=-332+1192 a b-492 a b^{2} \\
& c_{3}=-296(-5.03397+a b)(-0.425487+a b) \\
& c_{4}=-64(-22.0845+a b)(-0.540538+a b) \\
& c_{5}=-664+704 a b \\
& c_{6}=-376+136 a b \\
& c_{7}=-120 \\
& c_{8}=-16
\end{aligned}
$$

Now we have the following results:

\section{Theorem 1}

(i) The Hopf bifurcation for system(1) occurs at $\mu$ which is unique root of $g(\mu)$ under the condition $a b>0$ and $\sum_{i=0}^{8} c_{i}<0$.

(ii) There is no possibility of Hopf bifurcation if $a b=0$ or $a b<0$ along with $c_{1}, c_{2}, \ldots, c_{8}<0$, where $c_{i}$ s are given in equation (20).

Proof: Using Descarte's rule of sign results are easily verifiable. 


\section{Stability of bifurcating periodic solution}

To study the stability of bifurcating periodic solutions the frequency-domain formulation of Moiola and Chen(1996) is applied. By applying second order harmonic balance approximation to the output, we have

$$
y(t)=y^{*}+R\left(\sum_{k=0}^{2} Y_{k} e^{i k \omega t}\right)
$$

where $y^{*}$ is the equilibrium point, $\mathrm{R}$ is the real part of the complex constant, and the complex coefficients $Y_{k}$ are determined by the approximation as shown below: we define an auxiliary vector of the following form

$$
\xi_{1}(\tilde{\omega})=\frac{-\omega^{T}[G(\tilde{\omega} ; \tilde{\tau})] p_{1}}{\omega^{T} v}
$$

. where $\bar{\tau}$ is a fixed value of the parameter $\tau, \bar{\omega}$ is the frequency of the intersection between the $\bar{\lambda}(i \bar{\omega} ; \bar{\tau})$ locus and the negative real axis closest to the point $(-1+i 0), \omega^{T}$ and $\mathrm{v}$ are the left and right eigenvectors of $[G(i \bar{\omega} ; \bar{\tau})] J e^{-i \bar{\omega} \tau}$, respectively, associated with the value

$$
\bar{\lambda}(i \bar{\omega} ; \bar{\tau})
$$

and

$$
p_{1}=\left[D_{2}\left(V_{02} \otimes v+1 / 2 \bar{v} \otimes V_{22}\right)+1 / 8 D_{3} v \otimes v \otimes \bar{v}\right]
$$

where,

$$
\begin{gathered}
D_{2}=\frac{\partial^{2} g(y ; \tau)}{\partial y^{2}}, D_{3}=\frac{\partial^{3} g(y ; \tau)}{\partial y^{3}} \\
V_{02}=-\frac{1}{4}[I+G(0 ; \bar{\tau}) J(\bar{\tau})]^{-1} G(0 ; \bar{\tau}) D_{2} v \otimes \bar{v} \\
V_{22}=-\frac{1}{4}[I+G(2 i \bar{\omega} ; \bar{\tau}) J(\bar{\tau})]^{-1} G(2 i \bar{\omega} ; \bar{\tau}) D_{2} v \otimes v
\end{gathered}
$$

Then we have,

$$
D_{2}=[a(i, j)]_{3 \times 81}, D_{3}=[b(i, j)]_{3 \times 729}
$$

where non-zero elements of $D_{2}$ and $D_{3}$ are listed below:

$\mathrm{a}(1,1), \mathrm{a}(1,4), \mathrm{a}(1,7), \mathrm{a}(1,11), \mathrm{a}(1,14), \mathrm{a}(1,17), \mathrm{a}(1,21), \mathrm{a}(1,24), \mathrm{a}(1,27), \mathrm{a}(1,28), \mathrm{a}(1,31), \mathrm{a}(1,31)$, $\mathrm{a}(1,38), \mathrm{a}(1,41), \mathrm{a}(1,48), \mathrm{a}(1,51), \mathrm{a}(1,55), \mathrm{a}(1,65), \mathrm{a}(1,75)$,

$\mathrm{a}(2,1), \mathrm{a}(2,4), \mathrm{a}(2,7), \mathrm{a}(2,11), \mathrm{a}(2,14), \mathrm{a}(2,17), \mathrm{a}(2,28), \mathrm{a}(2,31), \mathrm{a}(2,38), \mathrm{a}(2,41), \mathrm{a}(2,55), \mathrm{a}(2,65)$,

$\mathrm{a}(3,1), \mathrm{a}(3,4), \mathrm{a}(3,7), \mathrm{a}(3,21), \mathrm{a}(3,24), \mathrm{a}(3,27), \mathrm{a}(3,28), \mathrm{a}(3,31), \mathrm{a}(3,48), \mathrm{a}(3,55), \mathrm{a}(3,75)$,

$\mathrm{b}(1,1), \mathrm{b}(1,4), \mathrm{b}(1,7), \mathrm{b}(1,28), \mathrm{b}(1,31), \mathrm{b}(1,55), \mathrm{b}(1,92), \mathrm{b}(1,95), \mathrm{b}(1,98)$,

$\mathrm{b}(1,119), \mathrm{b}(1,122), \mathrm{b}(1,135), \mathrm{b}(1,174), \mathrm{b}(1,177), \mathrm{b}(1,180)$ etc.

Theorem 2 (The graphical Hopf bifurcation theorem[10])Suppose that the locus of the distinguished characteristic function $\bar{\lambda}(s)$ intersects the negative real axis at $\bar{\lambda}(i \bar{\omega})$ that is closest to the point $(-1+\mathrm{i} 0)$ when the variable s sweeps on the classical Nyquist contour. Moreover, suppose that $\xi_{1}(\bar{\omega})$ is non zero and the half-line $L_{1}$ starting from $(-1+\mathrm{i} 0)$ and pointing to the direction parallel to that of $\xi_{1}(\bar{\omega})$, first intersects the locus of $\bar{\lambda}(i \omega)$ at

$$
\bar{\lambda}(i \omega)=\bar{P}=-1+\xi_{1}(\bar{\omega}) \bar{\theta}^{2},
$$

at which $\bar{\theta}=\theta(\bar{\omega}) \geq 0$ and $\theta=O\left(\left|\mu-\mu_{0}\right|^{1 / 2}\right)$. Finally, suppose that the following conditions are satisfied:

(i) The eigenlocus $\bar{\lambda}$ has nonzero rate of change with respect to its parametrization at the critical point $\left(\omega_{0}, \mu_{0}\right)$, that is,

$$
M\left(\omega_{0}, \mu_{0}\right)=\operatorname{det}\left[\begin{array}{ll}
\frac{\partial F_{1}}{\partial \mu} & \frac{\partial F_{2}}{\partial \mu} \\
\frac{\partial F_{1}}{\partial \omega} & \frac{\partial F_{2}}{\partial \omega}
\end{array}\right]_{\left(\omega_{0}, \mu_{0}\right)} \neq 0
$$


where

$$
F_{1}(\omega ; \mu)=\Re[h(-1, i \omega ; \mu)] .
$$

(ii) The intersection is transversal, namely,

$$
\operatorname{det}\left[\begin{array}{cc}
\Re\left[\xi_{1}(i \bar{\omega})\right] & \Im\left[\xi_{1}(i \bar{\omega})\right] \\
\left.\Re \frac{d}{d \omega} \bar{\lambda}(\omega)\right|_{\omega=\bar{\omega}} & \left.\Im \frac{d}{d \omega} \bar{\lambda}(\omega)\right|_{\omega=\bar{\omega}}
\end{array}\right] \neq 0
$$

(iii)There are no other intersections between any of the characteristic loci and the line segment joining the point $(-1+\mathrm{i} 0)$ to $\bar{P}$, at at least within a small neighborhood of radius $\delta>0$.

Then system (10) has a periodic solution $\mathrm{Y}(\mathrm{t})$ of frequency $\omega=\bar{\omega}+O\left(\bar{\theta}^{4}\right)$. Moreover, by applying a small perturbation around the intersection $\bar{P}$ and using the generalized Nyquist stability criterion, the stability of the periodic solution $\mathrm{Y}(\mathrm{t})$ can be determined.

According to theorem 2 we have the following conclusion:

If the total number of the point $P_{1}=\bar{P}+\epsilon \xi_{1}(\bar{\omega})$, for a small enough $\epsilon>0$, is equal to the number of poles of $\lambda(s)$ that have positive real parts, then the limit cycle is stable; otherwise, it is unstable.

According to eq. (15) we have

$$
\lambda^{2}(s)=\frac{2 a b\left[\left(\mu_{0}+s\right)^{2}-\mu_{0}^{2}\right]}{(1+s)^{2}\left(\mu_{0}+s\right)^{4}}
$$

Hence, $s=-1,-1,-\mu_{0},-\mu_{0},-\mu_{0},-\mu_{0}$ are poles of a $\lambda(s)$ that have positive real parts is zero.

Theorem 3 Let $\rho$ be the total number of anticlockwise encirclements of the point $P_{1}=\bar{P}+\epsilon \xi_{1}(\bar{\omega})$ for a small enough $\epsilon>0$, where $\bar{P}$ is the intersection of the half-line $L_{1}$ and the locus $\bar{\lambda}(i \omega)$. If $\rho=0$, then bifurcating periodic solutions of system (1) is stable and if $\rho \neq 0$, bifurcating periodic solutions of system (1) is unstable.

\section{Numerical examples}

In this section, some numerical simulations are given to verify Theorem 1 and stability of periodic solutions. The bifurcation diagram depicts how distributed delays play important role to stabilize the system.

Let us consider the three-neuron network model with distributed delay as follows

$$
\begin{aligned}
d y_{1}(t) / d t & =-y_{1}(t)+b\left[\tanh \left[y_{2}(t)-\int_{0}^{\infty} \mu^{2} s e^{-\mu s} y_{2}(t-s) d s\right]\right] \\
& +b\left[\tanh \left[y_{3}(t)-\int_{0}^{\infty} \mu^{2} s e^{-\mu s} y_{3}(t-s) d s\right]\right] \\
d y_{2}(t) / d t & =-y_{2}(t)+a\left[\tanh \left[y_{1}(t)-\int_{0}^{\infty} \mu^{2} s e^{-\mu s} y_{1}(t-s) d s\right]\right] \\
d y_{3}(t) / d t & =-y_{3}(t)+a\left[\tanh \left[y_{1}(t)-\int_{0}^{\infty} \mu^{2} s e^{-\mu s} y_{1}(t-s) d s\right]\right]
\end{aligned}
$$

we have following numerical simulations: 


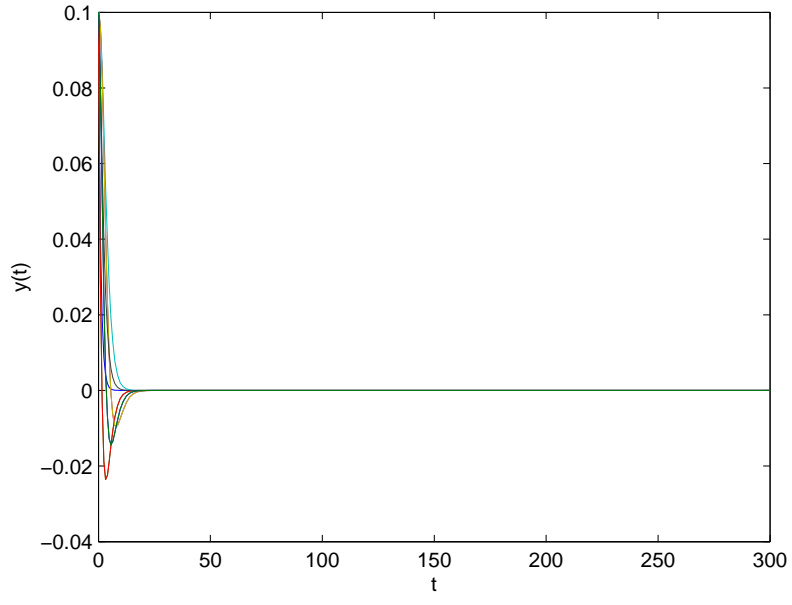

Figure 1: For $\mu=0.7, a=0.5, b=0$ origin is locally asymptotically stable.

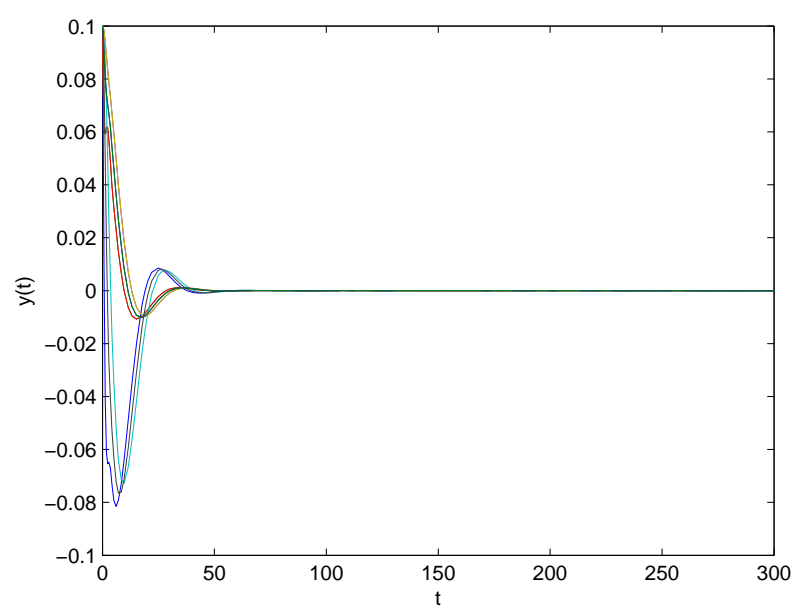

Figure 3: For $\mu=0.7, a=-0.5, b=1.5$ origin is locally asymptotically stable.

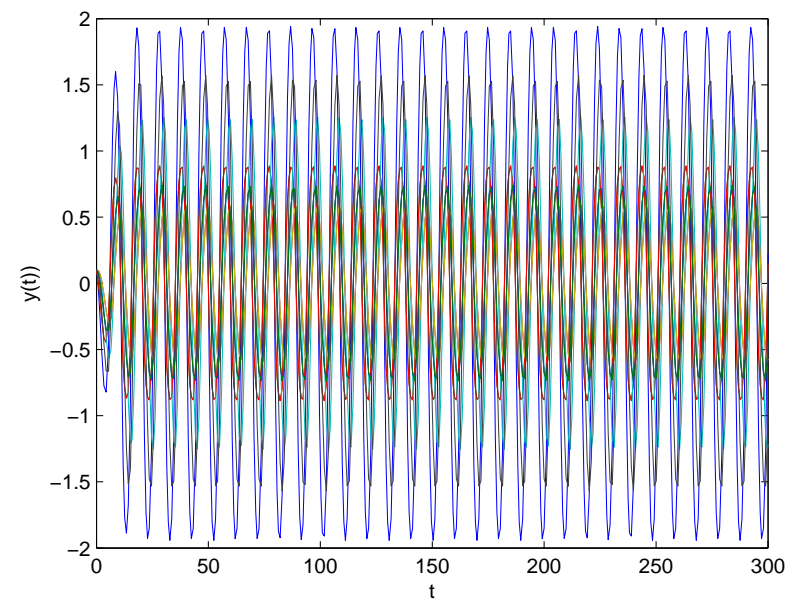

Figure 5: For $\mu=0.8, a=1, b=1.5$ time series depicts stable periodic oscillations.

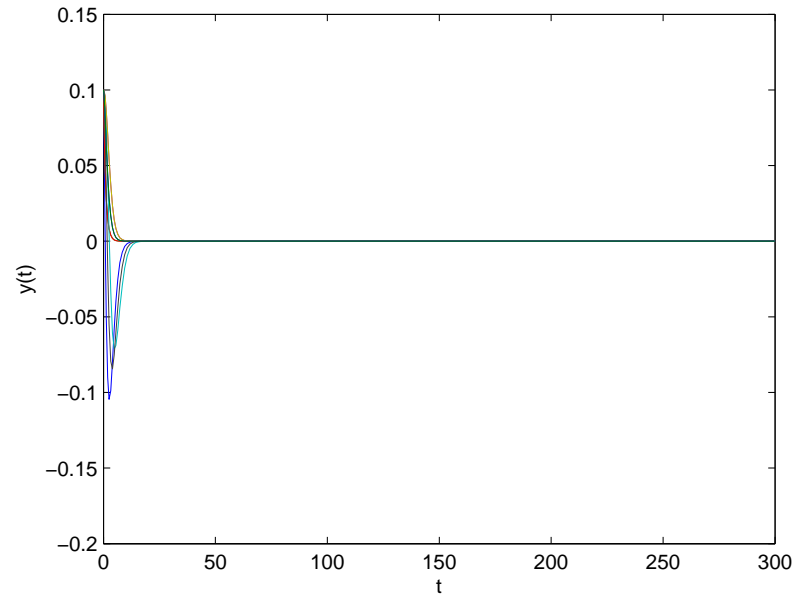

Figure 2: For $\mu=1, a=0, b=1.2$ origin is locally asymptotically stable.

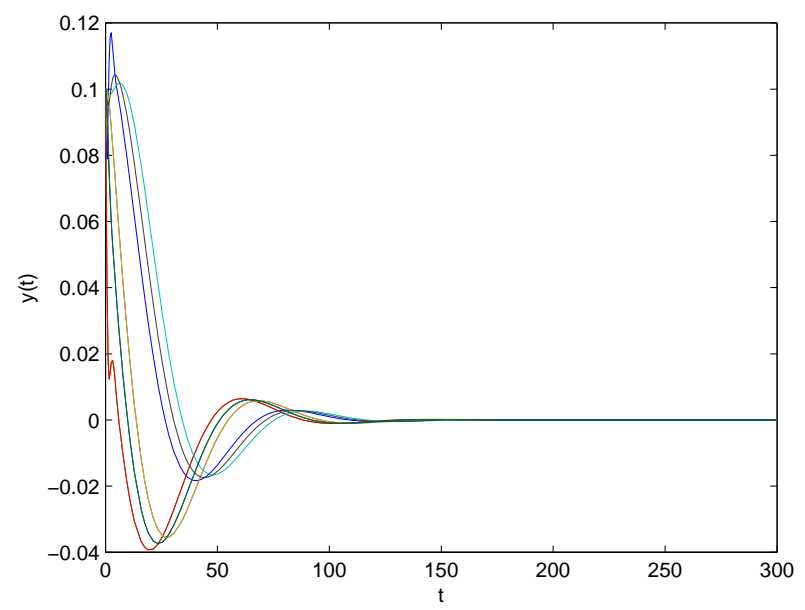

Figure 4: For $\mu=0.3, a=1.2, b=-0.8$ origin is locally asymptotically stable.

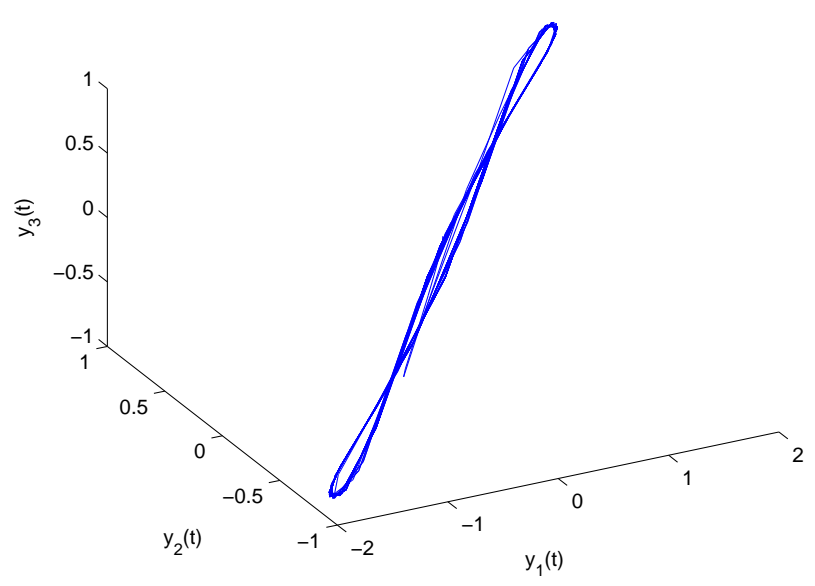

Figure 6: For $\mu=0.8, a=1, b=1.5$ phase portrait of $y_{1}, y_{2}, y_{3}$ shows stable limit cycle. 


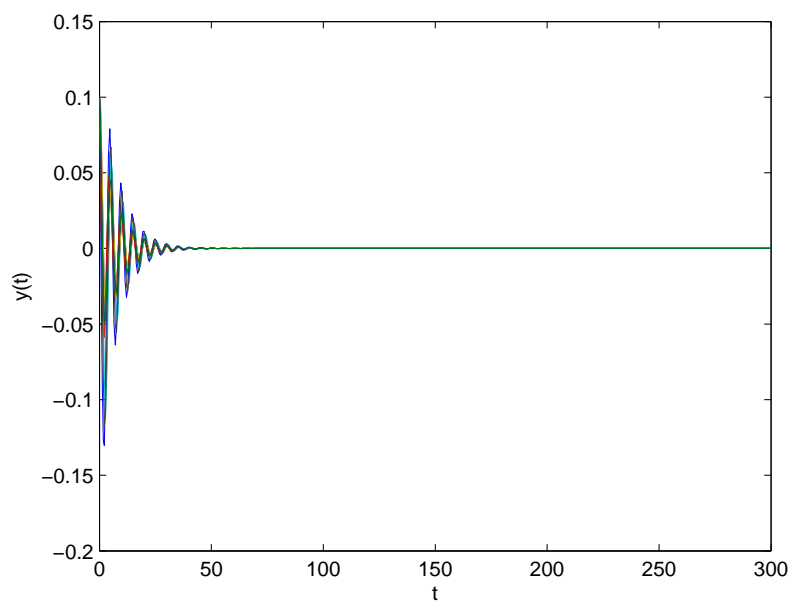

Figure 7: For $\mu=2.5, a=1, b=1.5$, when $\mu$ exceeds the critical value near 2.2 , origin becomes locally asymptotically stable.

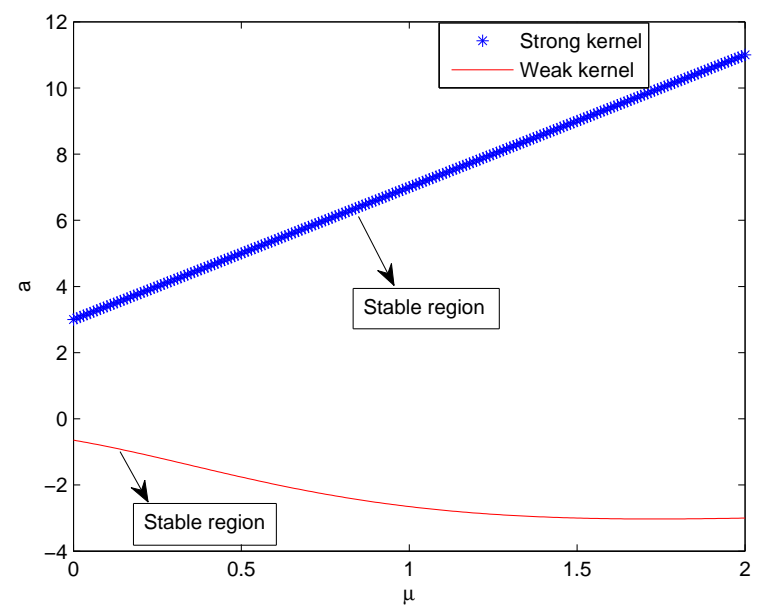

Figure 9: In $\mu-a$ parametric plane stability regime for strong kernel is enhanced than that for weak kernel when $\beta=0$.

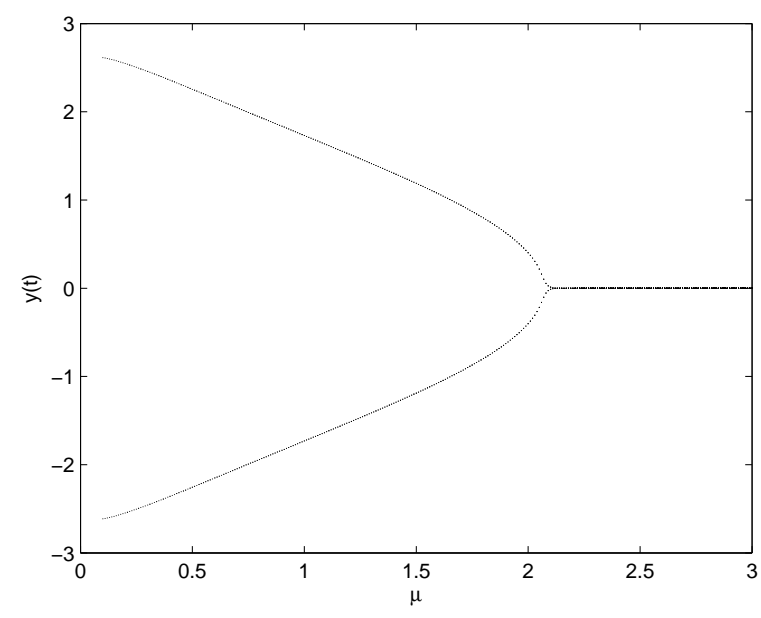

Figure 8: Bifurcation diagram, when bifurcation parameter $\mu$ varies.

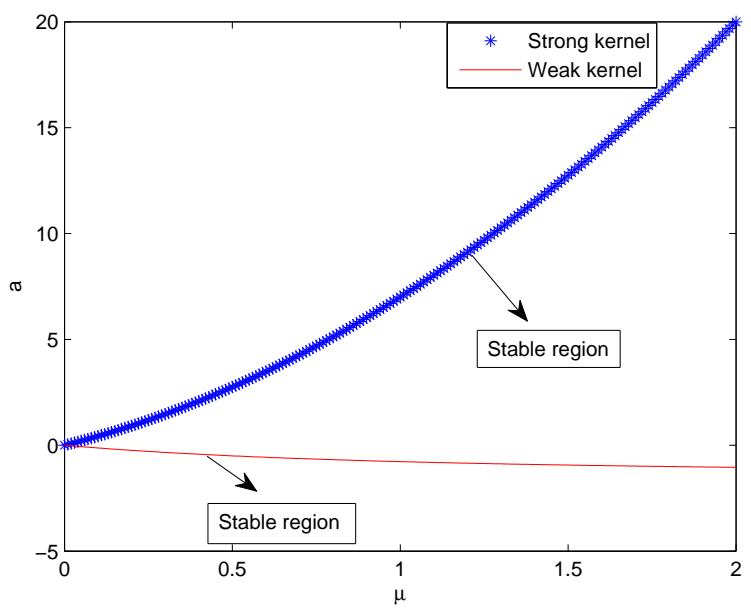

Figure 10: In $\mu-a$ parametric plane stability regime for strong kernel is enhanced than that for weak kernel when $\beta=$ 1.

\section{Conclusions}

With the help of the frequency domain approach we obtained the sufficient conditions for existence of Hopf bifurcation. It is seen that Hopf bifurcation occurs only when two connection strengths $a^{\prime}$ and $b^{\prime}$ are either both excitatory or inhibitory. Conditions are obtained for stability of Hopf bifurcating periodic solutions. One can determine the direction of Hopf bifurcation with the help of appropriate software, which can handle the matrix of order $3 \times 729$. The stabilizing effect of distributed delays is observed here. We have two graphical representations Fig.9 and Fig.10 in $\mu-a$ parametric plane, where the gain parameter $a$ is the product of connection strength $a^{\prime}$ and inclination of activation function $f$ at origin. In $\mu-a$ parametric plane stability regime for strong kernel is enhanced than that of the weak kernel. 


\section{Acknowledgements}

This Research work is supported and funded by TEQIP-II fund of JIS College of Engineering, Kalyani, India.

\section{References}

[1] F.M. Atay. "Distributed delays facilitate amplitude death of coupled oscillators", Phys. Rev. Lett., 91,(2003), pp.094-101.

[2] F.M. Atay. "Oscillator death in coupled functional differential equations near Hopf bifurcation", J. Diff. Eqs., 221,(2006), pp.190-209.

[3] V.K. Jirsa and M. Ding. "Will a large complex system with delays be stable?", Phys. Rev. Lett., 93,(2004), pp. 070602.

[4] C. W. Eurich, A. Thiel, L. Fahse," Distributed delays stabilize ecological feedback systems.", Phys. Rev. Lett., 94, 158104-1-4 (2005).

[5] U. Meyer,J. Shao,S. Chakrabarty,S. F. Brandt,H. Luksch,R. Wessel, "Distributed delays stabilize neural feedback systems.",Biological Cybernetics, 99,(2008) pp. 79-87.

[6] C. Zhang and X. Yan," Stability and hopf bifurcations in a delayed predator-prey system with a distributed delay", Int. J. Bifurcation Chaos, 19, 2283, (2009).

[7] S. Ruan ,"Delay differential equations in single species dynamics", Delay Differential Equations and Applications, Springer, Berlin,(2006),pp. 477-517.

[8] D. J. Allwright," Harmonic balance and the Hopf bifurcation theorem", Mathematical Proceedings of the Cambridge Philosophical Society, 82,(1977),pp. 453-467.

[9] A. I. Mees, L. O. Chua," The Hopf bifurcation theorem and its applications to nonlinear oscillations in circuits and systems," IEEE Transactions on Circulatory Systems, 26,(1979), pp.235-254.

[10] J. L. Moiola, G. R. Chen," Hopf bifurcation analysis: a frequency domain approach", Singapore: World Scientific(1996).

[11] A. I. Mees," Dynamics of feedback systems", John Wiley and Sons, Chichester, U.K.(1981). 